Mon. Not. R. Astron. Soc. 000, 000-000 (0000) Printed 2 May $2019 \quad$ (MN LATEX style file v2.2)

\title{
The g-mode spectrum of reactive neutron star cores
}

\author{
N. Andersson and P. Pnigouras \\ Mathematical Sciences and STAG Research Centre, University of Southampton, Southampton SO17 1BJ, UK
}

2 May 2019

\begin{abstract}
We discuss the impact of nuclear reactions on the spectrum of gravity g-modes of a mature neutron star, demonstrating the anticipated disappearance of these modes when the timescale associated with the oscillations is longer than that of nuclear reactions. This is the expected result, but different aspects of the demonstration may be relevant for related problems in neutron star astrophysics. In particular, we develop the framework required for an explicit implementation of finite-time nuclear reactions in neutron star oscillation problems and demonstrate how this formulation connects with the usual bulk viscosity prescription. We also discuss implications of the absence of very high order g-modes for problems of astrophysical relevance.
\end{abstract}

\section{INTRODUCTION}

Mature neutron stars support a family of gravity g-modes, that owe the buoyancy associated with a varying (with density) composition to their existence (Reisenegger \& Goldreich 1992). Similarly, hot young neutron stars may exhibit g-modes supported by entropy gradients (Ferrari et al (2003). However, the thermal buoyancy weakens as the star cools, leading to a gradual evolution of the g-mode spectrum (Krüger et al. 2014). Still, the g-modes of a hot neutron star may impact on gravitational-wave emission from the immediate aftermath of the core collapse in which the star was born (Ott et al. 2006). They may also be relevant for attempts to infer stellar parameters through asteroseismology. The g-modes that remain as the star cools down may also impact on observations. In particular, a number of studies have considered possible resonances between the g-modes and the tide induced by a binary partner, and to what extent resonant mode excitation may affect the gravitational-wave signal from an inspiralling neutron star binary (Kokkotas \& Schäfer 1995, Lai 1999, Andersson \& Ho 2018) - a topical issue following the spectacular GW170817 merger event (Abbott et al 2017).

The g-modes also enter the tidal problem in a somewhat unexpected way, as the high-order modes may couple nonlinearly to the tide and high-order pressure p-modes, leading to a non-resonant instability (Weinberg et al. 2013, Essick et al 2016). While this p-g instability is not yet fully understood, a search for its signature in the GW170817 data has been carried out [albeit with an inconclusive result, see Abbott et al (2019)]. Finally, the g-modes are important for the dynamics of isolated neutron stars. In particular, they are thought to be key to the saturation (again through nonlinear mode coupling) of the gravitational-wave driven instability of the fundamental f-mode in fast spinning stars (Pnigouras \& Kokkotas 2016), and they may play a similar role in the r-mode instability problem (Arras et al 2003). Given this, there has been some effort to understand the interplay between buoyancy and inertial effects in spinning neutron stars
(Passamonti et al 2009 Gaertig \& Kokkotas 2009). In particular, it has been demonstrated that the g-modes become dominated by the inertia at high rotation rates.

In essence, a detailed understanding of the g-mode spectrum of realistic neutron star models may be required for progress on a range of relevant astrophysics problems. Moreover, as the g-mode spectrum depends on both the matter composition and state of matter [with the presence of superfluid components being particularly significant, see Lee (1995); Andersson \& Comer (2001); Kantor \& Gusakov (2014); Passamonti et al. (2016)], it is interesting to ask to what extent one may be able to use observations to constrain high-density physics. This short note should be viewed in that context. Focussing on composition g-modes of cold neutron stars, we provide an explicit demonstration of the "disappearance" of g-modes with oscillation period longer than the timescale of the involved nuclear reactions. This result is not in any way unexpected. Nevertheless, we believe it is a worthwhile discussion. In particular, the (formal) absence of very low frequency g-modes helps explain the absence of tidal resonances in well separated binaries (a problem we will explore elsewhere). A cut-off in the highorder g-mode spectrum may also be relevant for nonlinear mode-coupling scenarios (e.g. relating to the $\mathrm{p}-\mathrm{g}$ instability or the saturation of unstable modes). Finally, our simple analysis highlights the resonant behaviour associated with bulk viscosity due to nuclear reactions. In fact, the way we set up the problem outlines an alternative to the standard bulk viscosity description, which may be useful for a wider range of problems (including numerical simulations).

\section{FORMULATING THE PROBLEM}

\subsection{Fluid perturbations}

As the main aim of our discussion is to provide a proof of principle, we focus on a relatively simple setting. In order to elaborate on the central question, we need to consider 
perturbations of a background star while keeping track of deviations from chemical equilibrium induced by the fluid motion. This introduces the relevant reaction timescales into the problem and allows us to consider the behaviour in different limits.

As we are dealing with local physics, it is natural to use a Lagrangian approach to the perturbation problem (Friedman \& Schutz 1978). Assuming that the star is non-rotating, we then first of all have the perturbed continuity equation (for the density $\rho$ )

$$
\partial_{t}\left(\Delta \rho+\rho \nabla_{i} \xi^{i}\right)=0
$$

where $\xi^{i}$ is the Lagrangian displacement vector associated with the perturbation

$$
\Delta=\delta+\mathcal{L}_{\xi}
$$

with $\delta$ the corresponding Eulerian perturbation (and $\mathcal{L}_{\xi}$ the Lie derivative along $\xi^{i}$ ) such that

$$
\Delta v^{i}=\delta v^{i}=\partial_{t} \xi^{i}
$$

The perturbed Euler equation is then

$$
\partial_{t}^{2} \xi_{i}+\frac{1}{\rho} \nabla_{i} \delta p-\frac{1}{\rho^{2}} \delta \rho \nabla_{i} p+\nabla_{i} \delta \Phi=0
$$

where $p$ is the fluid pressure and $\Phi$ is the gravitational potential.

We also have the Poisson equation for the perturbed gravitational potential

$$
\nabla^{2} \delta \Phi=4 \pi G \delta \rho
$$

while the unperturbed background configuration (assumed to be in hydrostatic equilibrium) is such that

$$
\nabla_{i} p=-\rho \nabla_{i} \Phi \equiv-\rho g_{i}
$$

where we have introduced the gravitational acceleration $g_{i}$, for later convenience.

\subsection{Adding reactions}

As we have already indicated, we are interested in the impact of nuclear reactions on the composition g-modes. As this involves keeping track of the matter composition, we take as our starting point a two-parameter equation of state $p=p\left(\rho, x_{\mathrm{p}}\right)$, where $x_{\mathrm{p}}=n_{\mathrm{p}} / n$ is the proton fraction. It is worth noting that, in the Newtonian case considered here, the mass density is simply $\rho=m_{B} n$ where $n$ is the baryon number density and $m_{B}$ is the baryon mass. Hence, we can think of $\rho$ as a proxy for the number density. Moreover, the continuity equation (1) remains unchanged (although, strictly speaking, it now represents baryon number conservation).

In order to account for nuclear reactions, we first of all introduce a new dependent variable $\beta=\mu_{\mathrm{n}}-\mu_{\mathrm{p}}-\mu_{\mathrm{e}}$ which encodes the deviation from chemical equilibrium (with $\mu_{\mathrm{x}}$, $\mathrm{x}=\mathrm{n}, \mathrm{p}$, e the chemical potentials for neutrons, protons and electrons, respectively). For simplicity, we assume a pure npe-matter neutron star core (cold enough that it is transparent to the neutrinos generated in the reactions), which means that the relevant reaction timescales are those associated with the Urca reactions. We then have

$$
d p=\sum_{\mathrm{x}} n_{\mathrm{x}} d \mu_{\mathrm{x}}=n\left(d \mu_{\mathrm{n}}-x_{\mathrm{p}} d \beta\right)
$$

where we have assumed (local) charge neutrality $\left(n_{\mathrm{p}}=n_{\mathrm{e}}\right)$. As the background configuration is in both hydrostatic and beta-equilibrium we only account for reactions at the level of the perturbations. To do this we, first of all, need to note that (7) should also hold at the (Lagrangian) perturbative level. That is, we have

$$
\Delta p=n\left(\Delta \mu_{\mathrm{n}}-x_{\mathrm{p}} \Delta \beta\right)
$$

or, as it turns out to be more convenient to work with $\rho$ and $\beta$,

$$
\begin{aligned}
\Delta p=\left(\frac{\partial p}{\partial \rho}\right)_{\beta} \Delta \rho+\left(\frac{\partial p}{\partial \beta}\right)_{\rho} & \Delta \beta \\
& =c_{s}^{2} \Delta \rho+\left(\frac{\partial p}{\partial \beta}\right)_{\rho} \Delta \beta
\end{aligned}
$$

where $c_{s}^{2}$ is sound speed of matter in chemical equilibrium.

The question is, what can we say about reactions? For the protons, we have (in general)

$$
\left(\partial_{t}+v^{j} \nabla_{j}\right) n_{\mathrm{p}}+n_{\mathrm{p}} \nabla_{i} v^{i}=\Gamma
$$

with $\Gamma$ the relevant reaction rate. Combining this with overall baryon number conservation

$$
\left(\partial_{t}+v^{j} \nabla_{j}\right) n+n \nabla_{i} v^{i}=0
$$

we get (assuming that protons and neutrons move together)

$$
\left(\partial_{t}+v^{j} \nabla_{j}\right) x_{\mathrm{p}}=\frac{\Gamma}{n}
$$

However, we assume that the reaction rate relates to perturbations. That is, we need

$$
\Delta\left[\left(\partial_{t}+v^{j} \nabla_{j}\right) x_{\mathrm{p}}\right]=\left(\partial_{t}+v^{j} \nabla_{j}\right) \Delta x_{\mathrm{p}}=\frac{\Gamma}{n}
$$

where, at least for small deviations from equilibrium (Haensel 1992, Reisenegger 1995),

$$
\Gamma \approx \gamma \Delta \beta
$$

with the $\gamma$ coefficient encoding the reaction rates.

Thinking of $\beta$ as a function of $\rho$ and $x_{\mathrm{p}}$, and assuming that the star is non-rotating (so that $v^{i}=0$ ), we have

$$
\partial_{t} \Delta \beta=\left(\frac{\partial \beta}{\partial \rho}\right)_{x_{\mathrm{p}}} \partial_{t} \Delta \rho+\left(\frac{\partial \beta}{\partial x_{\mathrm{p}}}\right)_{\rho} \partial_{t} \Delta x_{\mathrm{p}}
$$

which, once we use 13 , becomes

$$
\partial_{t} \Delta \beta=\left(\frac{\partial \beta}{\partial \rho}\right)_{x_{\mathrm{p}}} \partial_{t} \Delta \rho+\left(\frac{\partial \beta}{\partial x_{\mathrm{p}}}\right)_{\rho} \frac{\gamma}{n} \Delta \beta
$$

That is, we have

$$
\partial_{t} \Delta \beta-\mathcal{A} \Delta \beta=\mathcal{B} \partial_{t} \Delta \rho
$$

with

$$
\mathcal{A}=\left(\frac{\partial \beta}{\partial x_{\mathrm{p}}}\right)_{\rho} \frac{\gamma}{n}, \quad \mathcal{B}=\left(\frac{\partial \beta}{\partial \rho}\right)_{x_{\mathrm{p}}}
$$

The coefficients $\mathcal{A}$ and $\mathcal{B}$ should be time independent, so if we work in the frequency domain (essentially assuming a time-dependence $e^{i \omega t}$ for the perturbations, without introducing specific notation for the Fourier amplitudes) then we have

$$
\Delta \beta=\frac{\mathcal{B}}{1+i \mathcal{A} / \omega} \Delta \rho
$$


Let us now consider the timescales involved. Introducing a characteristic reaction time as

$$
t_{R}=\frac{1}{\mathcal{A}}
$$

(noting that the actual timescale is the absolute value of this) we see that, if the reactions are fast compared to the dynamics (on a timescale $\sim 1 / \omega$ ) then $\left|t_{R} \omega\right| \ll 1$ and we have

$$
\Delta \beta \approx 0 .
$$

Basically, the fluid remains in beta-equilibrium.

However, in the limit of slow reactions we have $\left|t_{R} \omega\right| \gg$ 1 and we can Taylor expand $\sqrt{19}$ to get

$$
\Delta \beta \approx \mathcal{B}(1-i \mathcal{A} / \omega) \Delta \rho \approx \mathcal{B} \Delta \rho
$$

Using this result in $(9)$, we have

$$
\Delta p=\left[\left(\frac{\partial p}{\partial \rho}\right)_{\beta}+\left(\frac{\partial p}{\partial \beta}\right)_{\rho}\left(\frac{\partial \beta}{\partial \rho}\right)_{x_{\mathrm{p}}}\right] \Delta \rho \equiv \mathcal{C} \Delta \rho
$$

which leads to

$$
\delta p=\mathcal{C} \delta \rho+\left[\mathcal{C} \xi^{j} \nabla_{j} \rho-\xi^{j} \nabla_{j} p\right]
$$

However, since

$$
\nabla_{j} p=\left(\frac{\partial p}{\partial \rho}\right)_{\beta} \nabla_{j} \rho=c_{s}^{2} \nabla_{j} \rho
$$

we are left with

$$
\delta p=\mathcal{C} \delta \rho+\left(\frac{\partial p}{\partial \beta}\right)_{\rho}\left(\frac{\partial \beta}{\partial \rho}\right)_{x_{\mathrm{p}}} \xi^{j} \nabla_{j} \rho
$$

The composition of matter impacts on both terms on the right-hand side of this relation.

Given an actual supranuclear equation of state, the different thermodynamical derivatives required to make the relation 26 explicit should be calculable [although we obviously need to start from a model that does not assume chemical equilibrium from the outset, see for example Passamonti et al. (2016)].

Before we proceed, let us confirm that the relations for the slow-reaction case correspond to frozen composition. This serves as a useful "sanity check" as it ensures that the mathematics agrees with intuition. We have (first thinking of the equation of state as $p=p(\rho, \beta)$ and then changing to $\left.p=p\left(\rho, x_{\mathrm{p}}\right)\right)$

$$
\begin{gathered}
\Delta p=\left(\frac{\partial p}{\partial \rho}\right)_{\beta} \Delta \rho+\left(\frac{\partial p}{\partial \beta}\right)_{\rho} \Delta \beta \\
=\left(\frac{\partial p}{\partial \rho}\right)_{\beta} \Delta \rho+\left(\frac{\partial p}{\partial \beta}\right)_{\rho}\left[\left(\frac{\partial \beta}{\partial \rho}\right)_{x_{\mathrm{p}}} \Delta \rho+\left(\frac{\partial \beta}{\partial \mathrm{x}_{\mathrm{p}}}\right)_{\rho} \Delta x_{\mathrm{p}}\right] \\
=\left[\left(\frac{\partial p}{\partial \rho}\right)_{\beta}+\left(\frac{\partial p}{\partial \beta}\right)_{\rho}\left(\frac{\partial \beta}{\partial \rho}\right)_{x_{\mathrm{p}}}\right] \Delta \rho \\
+\left(\frac{\partial p}{\partial \beta}\right)_{\rho}\left(\frac{\partial \beta}{\partial \mathrm{x}_{\mathrm{p}}}\right)_{\rho} \Delta x_{\mathrm{p}} \\
=\mathcal{C} \Delta \rho+\left(\frac{\partial p}{\partial \mathrm{x}_{\mathrm{p}}}\right)_{\rho} \Delta x_{\mathrm{p}}
\end{gathered}
$$

which means that, if 23 holds we must have $\Delta x_{\mathrm{p}}=0$. We also see that

$$
\left(\frac{\partial p}{\partial \rho}\right)_{x_{\mathrm{p}}}=\left(\frac{\partial p}{\partial \rho}\right)_{\beta}+\left(\frac{\partial p}{\partial \beta}\right)_{\rho}\left(\frac{\partial \beta}{\partial \rho}\right)_{x_{\mathrm{p}}}=\mathcal{C}
$$

This relation will prove useful later.

\section{THE G-MODE(S)}

Let us focus on a "toy version" of the g-mode problem. Starting from (4), i.e.

$$
-\omega^{2} \xi_{i}+\frac{1}{\rho} \nabla_{i} \delta p-\frac{1}{\rho^{2}} \delta \rho \nabla_{i} p+\nabla_{i} \delta \Phi=0
$$

we first of all make the Cowling approximation (neglect the perturbed gravitational potential $\delta \Phi)$, to get

$$
-\omega^{2} \xi_{i}+\frac{1}{\rho}\left(\nabla_{i} \delta p+g_{i} \delta \rho\right)=0
$$

Next we assume a plane-wave solution such that $\delta p \sim$ $e^{i k_{i} x^{i}}$ (and similar for all other variables). This leaves us with

$$
-\omega^{2} \xi_{i}+\frac{1}{\rho}\left(i k_{i} \delta p+g_{i} \delta \rho\right)=0
$$

\subsection{Fast reactions}

Now let us consider two limiting cases. First, for fast reactions we have seen that

$$
\Delta \beta=0 \longrightarrow \Delta p=c_{s}^{2} \Delta \rho
$$

or

$$
\delta p=c_{s}^{2} \delta \rho+\xi^{j}\left(c_{s}^{2} \nabla_{j} \rho-\nabla_{j} p\right)=c_{s}^{2} \delta \rho
$$

That is, we have

$$
-\omega^{2} \xi_{i}+\frac{1}{\rho}\left(i c_{s}^{2} k_{i}+g_{i}\right) \delta \rho=0
$$

However, the continuity equations leads to

$$
\begin{aligned}
\delta \rho=-\xi^{j} & \nabla_{j} \rho-\rho \nabla_{j} \xi^{j} \\
& =-\frac{1}{c_{s}^{2}} \xi^{j} \nabla_{j} p-i \rho k_{j} \xi^{j}=\frac{\rho}{c_{s}^{2}} g_{j} \xi^{j}-i \rho k_{j} \xi^{j}
\end{aligned}
$$

so we have

$$
-\omega^{2} \xi_{i}+\left(i c_{s}^{2} k_{i}+g_{i}\right)\left(\frac{1}{c_{s}^{2}} g_{j}-i k_{j}\right) \xi^{j}=0
$$

Contracting with the wave vector, we get

$$
-\omega^{2}\left(k_{i} \xi^{i}\right)+\left[i c_{s}^{2} k^{2}+\left(g_{i} k^{i}\right)\right]\left[\frac{1}{c_{s}^{2}}\left(g_{j} \xi^{j}\right)-i\left(k_{j} \xi^{j}\right)\right]=0
$$

It is useful to simplify this by assuming short wavelengths. Taking $k \gg g / c_{s}^{2}$ we get

$$
\left[\omega^{2}-c_{s}^{2} k^{2}\right]\left(k_{j} \xi^{j}\right) \approx 0
$$

That is, as long as $k_{j} \xi^{j} \neq 0$, we have the sound waves

$$
\omega \approx \pm c_{s} k
$$

The transverse solution, $k_{j} \xi^{j}=0$, is trivial. There are no g-modes in this case. 


\subsection{Slow reactions}

The case of slow reactions is a little bit more involved. Starting from

$$
\Delta p=\left(\frac{\partial p}{\partial \rho}\right)_{x_{\mathrm{p}}} \Delta \rho=\mathcal{C} \Delta \rho
$$

we find that

$$
\begin{aligned}
\delta \rho= & \frac{1}{\mathcal{C}} \delta p+\xi^{j}\left[\frac{1}{\mathcal{C}} \nabla_{j} p-\nabla_{j} \rho\right] \\
& =\frac{1}{\mathcal{C}} \delta p-\rho\left(g_{j} \xi^{j}\right)\left[\frac{1}{\mathcal{C}}-\frac{1}{c_{s}^{2}}\right]=\frac{1}{\mathcal{C}} \delta p+\rho\left(A_{j} \xi^{j}\right)
\end{aligned}
$$

where we have introduced

$$
A_{i}=\left[\frac{1}{c_{s}^{2}}-\frac{1}{\mathcal{C}}\right] g_{i}
$$

The radial component

$$
A=-g\left[\frac{1}{c_{s}^{2}}-\frac{1}{\mathcal{C}}\right]
$$

defines the Schwarzschild discriminant. We also need to use the continuity equation, to get

$$
\begin{aligned}
\delta p=\mathcal{C} \Delta & \rho-\xi^{j} \nabla_{j} p \\
& =-\rho \mathcal{C} \nabla_{j} \xi^{j}-\xi^{j} \nabla_{j} p=-\rho \mathcal{C} \nabla_{j} \xi^{j}+\rho\left(g_{j} \xi^{j}\right)
\end{aligned}
$$

In terms of plane waves, this means that

$$
\delta p=-i \rho \mathcal{C}\left(k_{j} \xi^{j}\right)+\rho\left(g_{j} \xi^{j}\right)
$$

Moving on to the Euler equation, we still have (34) but this now leads to

$$
\begin{aligned}
-\omega^{2} \xi_{i}+\left[i k_{i}+\frac{1}{\mathcal{C}} g_{i}\right]\left[-i \mathcal{C}\left(k_{j} \xi^{j}\right)+\right. & \left.\left(g_{j} \xi^{j}\right)\right] \\
& +g_{i}\left(A_{j} \xi^{j}\right)=0
\end{aligned}
$$

We create two scalar equations by contracting with $g^{i}$ and $k^{i}$, respectively. This leads to

$$
\begin{aligned}
\left\{-\omega^{2}-i \mathcal{C}\left[i k^{2}+\frac{1}{\mathcal{C}}\left(g_{j} k^{j}\right)\right]\right\}\left(k_{j} \xi^{j}\right) & \\
+ & {\left[i k^{2}+\frac{1}{c_{s}^{2}}\left(g_{j} k^{j}\right)\right]\left(g_{j} \xi^{j}\right)=0 }
\end{aligned}
$$

and

$$
\begin{aligned}
-i \mathcal{C}\left[i\left(k_{j} g^{j}\right)\right. & \left.+\frac{1}{\mathcal{C}} g^{2}\right]\left(k_{j} \xi^{j}\right) \\
& +\left[-\omega^{2}+\frac{1}{c_{s}^{2}} g^{2}+i\left(k_{j} g^{j}\right)\right]\left(g_{j} \xi^{j}\right)=0
\end{aligned}
$$

In order for this system to have solutions, we must have

$$
\begin{array}{r}
\left\{\omega^{2}-\mathcal{C}\left[k^{2}-\frac{i}{\mathcal{C}}\left(g_{j} k^{j}\right)\right]\right\}\left[\omega^{2}-\frac{1}{c_{s}^{2}} g^{2}-i\left(k_{j} g^{j}\right)\right] \\
-\left[k^{2}-\frac{i}{c_{s}^{2}}\left(g_{j} k^{j}\right)\right]\left[g^{2}+i \mathcal{C}\left(k_{j} g^{j}\right)\right]=0
\end{array}
$$

Let us simplify this by assuming that $g_{j} k^{j}=0$ (which does not change the qualitative nature of the solutions). Then we have

$$
\omega^{4}-\left(\mathcal{C} k^{2}+\frac{1}{c_{s}^{2}} g^{2}\right) \omega^{2}-\mathcal{C} k^{2} g A=0
$$

For short wavelengths (as before) this simplifies to

$$
\omega^{4}-\mathcal{C} k^{2} \omega^{2}-\mathcal{C} k^{2} g A=0
$$

and we have two sets of modes. For high frequencies, we get the sound waves

$$
\omega^{2} \approx \mathcal{C} k^{2}
$$

where it is worth noting that the matter composition has a (likely small, but nevertheless) effect on the mode frequency. Meanwhile, the low-frequency modes are given by

$$
\omega^{2}=-g A=N^{2}
$$

where $N$ is the usual Brunt-Väisälä frequency. This solution represents the composition g-modes (Reisenegger \& Goldreich 1992).

\subsection{The general case}

Suppose we now want to account for finite reaction times. Then the calculation from the previous sections goes through - pretty much unchanged - up to equation (51). However, as we want to make use the general result 119, , we need to replace $\mathcal{C}$ by

$$
\mathcal{D}=c_{s}^{2}+\frac{\omega \mathcal{B}}{\omega+i \mathcal{A}}\left(\frac{\partial p}{\partial \beta}\right)_{\rho}
$$

In order to do this, we need additional thermodynamical derivatives. However, we can use (28) to get

$$
\mathcal{B}\left(\frac{\partial p}{\partial \beta}\right)_{\rho}=\mathcal{C}-c_{s}^{2}
$$

That is, we have

$$
\mathcal{D}=c_{s}^{2}+\frac{\omega}{\omega+i \mathcal{A}}\left(\mathcal{C}-c_{s}^{2}\right)
$$

Introducing the reaction time, $t_{R}=1 / \mathcal{A}$ (as before), we have

$$
\mathcal{D}=\frac{1}{\omega t_{R}+i} \mathcal{C}\left[\omega t_{R}+i \frac{c_{s}^{2}}{\mathcal{C}}\right]
$$

so we need to solve

$$
\omega^{4}-\mathcal{D} k^{2} \omega^{2}-\mathcal{D} k^{2} g A=0
$$

with $A$ now given by

$$
A=-g\left[\frac{1}{c_{s}^{2}}-\frac{1}{\mathcal{D}}\right]
$$

It is also useful to note that

$$
\mathcal{D} k^{2} g A=-k^{2} \mathcal{C} g^{2} \frac{\omega t_{R}}{\omega t_{R}+i}\left(\frac{1}{c_{s}^{2}}-\frac{1}{\mathcal{C}}\right)
$$

While it would be straightforward to solve the problem for a specific stellar model, we prefer to illustrate the involved principles in terms of a suitably simply model problem. Thus we parameterise in terms of the mode frequencies in the slow reaction limit:

$$
\omega_{f}^{2}=\mathcal{C} k^{2}
$$

and

$$
\omega_{g}^{2}=g^{2}\left[\frac{1}{c_{s}^{2}}-\frac{1}{\mathcal{C}}\right]
$$


Note that, as we can vary $g$ here, the link to $\mathcal{C}$ and $c_{s}^{2}$ is not immediate. However, we know that we must have $\mathcal{C}>c_{s}^{2}$ in order to have oscillatory modes, and we can easily make sure that our model satisfies this constraint. We will also use the non-stratified result

$$
\omega_{0}^{2}=c_{s}^{2} k^{2}
$$

In terms of these parameters, we have

$$
\mathcal{D} k^{2}=\frac{1}{\omega t_{R}+i} \omega_{f}^{2}\left[\omega t_{R}+i \frac{\omega_{0}^{2}}{\omega_{f}^{2}}\right]
$$

and

$$
\mathcal{D} k^{2} g A=-\frac{\omega t_{R}}{\omega t_{R}+i} \omega_{f}^{2} \omega_{g}^{2}
$$

Finally, let us scale the frequencies to the acoustic mode, by introducing $x=\omega / \omega_{f}, \tilde{\omega}_{0}=\omega_{0} / \omega_{f}$ and $\tilde{\omega}_{g}=\omega_{g} / \omega_{f}$. Similarly parameterising the reaction time (through its relation to the g-mode frequency)

$$
t_{R}=\frac{\alpha}{\omega_{g}} \longrightarrow \omega t_{R}=\frac{\alpha x}{\tilde{\omega}_{g}} \quad \text { with } \quad \alpha<0
$$

(where the sign of $\alpha$ ensures that the modes of the toy problem are damped), we arrive at the final polynomial

$$
x^{4}-\frac{\tilde{\omega}_{g}}{\alpha x+i \tilde{\omega}_{g}}\left[\frac{\alpha x}{\tilde{\omega}_{g}}+i \tilde{\omega}_{0}^{2}\right] x^{2}+\frac{\alpha x}{\alpha x+i \tilde{\omega}_{g}} \tilde{\omega}_{g}^{2}=0
$$

The solutions to this problem illustrate the main principles, we are interested in. The top panel of figure 1 demonstrates the disappearance of the g-mode once the reaction timescale is shorter than that of the mode oscillation (when $\left.\left|t_{R} \omega_{g}\right| \lesssim 1\right)$. We also see that, at least in this model problem, the impact of the matter composition on the frequency of the sound waves is small. Meanwhile, the bottom panel of figure 1 illustrates how the damping of two sets of modes changes as we vary the the reaction time. The sharp disappearance of the oscillating g-mode when the imaginary part of the mode equals the real part is notable.

\subsection{Recovering bulk viscosity}

At this point it is relevant to note that, in addition to demonstrating the disappearance of "slow" composition gmodes, our simple model problem highlights the resonant behaviour associated with bulk viscosity (apparent in the fmode damping in figure 1). This is not surprising since reactions determine the rate of bulk viscosity in mature neutron stars [see, for example, Alford et al (2010)]. Nevertheless, our calculation provides an useful demonstration that the effect can be accounted for by explicitly allowing for the different particle fractions to evolve. In the extension, this approach could prove useful in nonlinear studies, where the deviation from chemical equilibrium is sufficiently large that the usual linearised approach cannot be applied. This may be particularly interesting given recent efforts to account for bulk viscosity in simulations of neutron star mergers (Alford et al 2018) and the parallel development of a theoretical framework that would (at least in principle) allow us to account for non-conserved particle flows (Andersson et al 2017).

As it may be instructive to recover the standard prescription from the present formulation, note that the slow
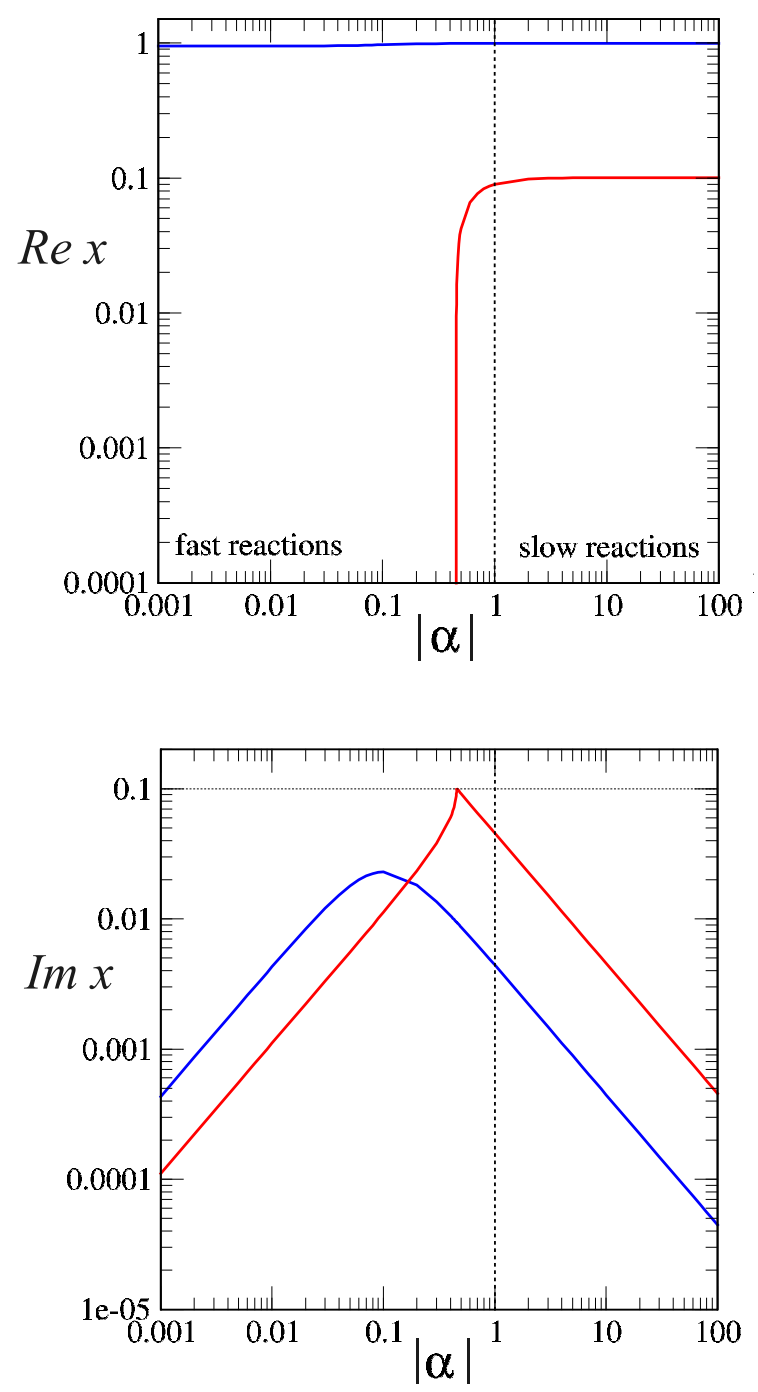

Figure 1. An illustration of the transition from the fast to the slow reaction regime and the impact on the relevant roots to the polynomial 67, representing acoustic modes (blue) and composition gravity modes (red). Top panel: We show the real parts of the mode frequencies for $\tilde{\omega}_{0}=0.95$ and $\tilde{\omega}_{g}=0.1$ as functions of the (scaled) reaction time $\alpha$. Bottom panel: The corresponding imaginary parts. The vertical dashed line in each panel indicates where $\left|t_{R} \omega_{g}\right|=1$, the point at which the g-mode oscillation period is equal to the reaction rate. The results demonstrate the expected disappearance of the g-mode for fast reaction rates. The sharp disappearance of the oscillating g-mode (roughly) when the imaginary part of the mode equals the real part (indicated by the horisontal dashed line) is notable. The results also bring out the expected resonance associated with the bulk viscosity damping of the acoustic mode.

reaction expansion of $(22)$ allows us to write $(9)$ as

$$
\begin{aligned}
& \Delta p \approx c_{s}^{2} \Delta \rho+\left(\frac{\partial p}{\partial \beta}\right)_{\rho} \mathcal{B}\left(1-i \frac{\mathcal{A}}{\omega}\right) \Delta \rho \\
&=\mathcal{C} \Delta \rho+i \frac{\mathcal{A}}{\omega}\left(\frac{\partial p}{\partial \beta}\right)_{\rho}\left(\frac{\partial \beta}{\partial \rho}\right)_{x_{\mathrm{p}}} \Delta \rho \\
&=\mathcal{C} \Delta \rho-\zeta \nabla_{j} \xi^{j}
\end{aligned}
$$


where

$$
\zeta=i \frac{\rho \mathcal{A}}{\omega}\left(\frac{\partial p}{\partial \beta}\right)_{\rho}\left(\frac{\partial \beta}{\partial \rho}\right)_{x_{\mathrm{p}}}
$$

In order to use this result in the Euler equation, we may introduce

$$
\delta p=\delta \bar{p}-\zeta \nabla_{j} \xi^{j}
$$

such that $\delta \bar{p}$ represents the "inviscid" pressure perturbation. This immediately leads to

$$
-\omega^{2} \xi_{i}+\frac{1}{\rho} \nabla_{i} \delta \bar{p}-\frac{1}{\rho^{2}} \delta \rho \nabla_{i} p+\nabla_{i} \delta \Phi=\nabla_{i}\left(\zeta \nabla_{j} \xi^{j}\right)
$$

where we recognize the term on the right-hand side as the bulk viscosity.

\section{IMPLICATIONS}

We have demonstrated that nuclear reactions may remove composition g-modes from the oscillation spectrum of a mature neutron star. This result should come as no surprise. The transition from individual g-modes being present to them being absent may be a bit sharper than expected, but the overall behaviour is intuitive. Nevertheless, there are valuable lessons to be learned. In particular, one may want to be a bit careful with assumptions involving highorder (very low frequency) g-modes. Two topical scenarios spring to mind: i) the saturation of modes driven unstable by gravitational-wave emission is thought to rely on the coupling to short-range (high overtone) modes (Pnigouras \& Kokkotas 2016), and ii) the tidal coupling to a pair of high overtone pressure p-modes and g-modes may lead to a non-resonant instability (Weinberg et al. 2013, Essick et al 2016). In both cases, the outcome may be affected (at least in principle) by the removal of g-modes from the lowfrequency spectrum.

As a guide, let us consider the problem for a hot young neutron star for which the radiation driven instability may be particularly relevant ${ }^{1}$. To get a rough idea, we may use the estimate timescales from Yakovlev et al (2001). The relevant equilibration timescales are ther ${ }^{2}$

$$
t_{M} \sim \frac{2 \text { months }}{T_{9}^{6}}, \quad t_{D} \sim \frac{20 \mathrm{~s}}{T_{9}^{4}}
$$

for the modified and direct Urca reactions, respectively. The temperature is scaled to relatively hot systems, $T_{9}=$ $T / 10^{9} \mathrm{~K}$. Taking $T_{9}=10$ for a typical proto-neutron star and assuming that g-modes disappear below a frequency

$$
\omega^{\text {cut }}=2 \pi f^{\text {cut }} \approx \frac{1}{t_{R}}
$$

in accord with the discussion in the previous section, we see that the spectrum would be altered below

$$
f_{M}^{\text {cut }} \sim 3 \times 10^{-2} \mathrm{~Hz}, \quad f_{D}^{\text {cut }} \sim 80 \mathrm{~Hz}
$$

1 We will consider the tidal problem elsewhere, as it requires a more in depth discussion.

2 Given that we have assumed npe-matter we do not consider the, significantly faster, reactions associated with hyperons. Nevertheless, it is easy to see what the implications for that problem would be. in the two cases. Assuming that the g-modes reside at frequencies below (say) $100 \mathrm{~Hz}$, we see that only a few g-modes may be allowed in a hot star in which the direct Urca channel is open, with additional modes entering the spectrum as the star cools. In contrast, in the standard case of modified Urca reactions, there should be a large number of g-modes already at high temperatures, but very high overtone modes can still not exist.

What do we learn from this? The conclusion may be more conceptual than of direct astrophysical relevance, but it is clear that one has to execute some level of care in discussions involving the dynamics of very high order g-modes. It is also relevant to consider how other aspects of neutron star physics enter the discussion. Superfluidity may be particularly important. After all, we know that superfluidity completely removes the g-modes for npe matter [as the superfluid neutrons may move relative to the charged components, see Lee (1995); Andersson \& Comer (2001)]. However, it is also known that the appearance of muons introduces relevant composition variation (now associated with the muon to electron ratio), which leads to the appearance of a set of (slightly higher frequency) g-modes (Kantor \& Gusakov 2014, Passamonti et al. 2016). These modes should, in principle, be affected by nuclear reactions. However, the neutron superfluidity also suppresses any reactions in which they are involved, which naturally affects the predicted cutoff frequency in the g-mode spectrum. Again, the outcome depends on the details.

Finally, it is worth noting that our analysis was based on the assumption that the deviation from chemical equilibrium was sufficiently small that we could linearise the problem. This should be a valid assumption for many problems of interest, but one can easily think of situations where nonlinear aspects come into play (like neutron star mergers). It is well known that nonlinear deviations from equilibrium lead to shorter equilibration times (Haensel 1992 , Reisenegger 1995, Alford et al 2010). As this may have a significant effect on any estimated g-mode cut-off frequency, it is a problem worth further consideration.

\section{ACKNOWLEDGEMENTS}

Support from STFC via grant ST/R00045X/1 is gratefully acknowledged.

\section{REFERENCES}

Abbott B. P., et al, 2017, Phys. Rev. Lett., 119, 161101

Alford M. G., Mahmoodifar S., Schwenzer K., 2012, J. Phys. G, 37, 125202

Alford M. G., Bovard L., Hanauske M., Rezzolla L., Schwenzer K, 2018, Phys. Rev. Lett. 120, 041101

Abbott B. P., et al, 2019, Phys. Rev. Lett., 122, 061104

Andersson N., Comer G. L., 2001, MNRAS, 328, 1129

Andersson N., Ho W. C. G., 2018, Phys. Rev. D, 97, 023016

Andersson N., Dionysopoulou K., Hawke I. Comer G. L., 2017, Class. Quantum Grav. 34, 125002

Arras P., et al, 2003, Ap. J., 591,1129

Essick R., Vitale S., Weinberg N. N., 2016, Phys. Rev. D, 94, 103012 
Ferrari V., Miniutti G., Pons J. A., 2003, Class. Quantum Grav. 20, S841

Friedman J. L., Schutz B. F., 1978, Ap, J., 221, 937

Gaertig E., Kokkotas K. D., 2009, Phys. Rev. D, 80, 064026

Haensel P., 1992, Astron. Astrophys., 262, 131

Kantor E. M., Gusakov M. E., 2014, MNRAS, 442, L90

Kokkotas K. D., Schäfer G., 1995, MNRAS, 275, 301

Krüger C. J., Ho W. C. G., Andersson N., 2015, Phys. Rev. D, 92, 063009

Lai D., 1999, MNRAS, 307, 1001

Lee U., 1995, Astron. Astrop., 303, 515

Ott C. D., Burrows A., Dessart L., Livne E., 2006, Phys. Rev. Lett., 96, 201102

Passamonti A., et al, 2009, MNRAS, 394, 730

Passamonti A., Andersson N., Ho W. C. G., 2016, MNRAS, 455,1489

Pnigouras P., Kokkotas K. D., 2016, Phys. Rev. D, 94, 024053

Reisenegger A., Goldreich P., 1992, Ap. J., 395, 240

Reisenegger A., 1995, Ap. J., 442, 749

Weinberg N. N., Arras P., Burkart J., 2013, Ap. J., 769, 121

Yakovlev D. G., Kaminker A. D., Gnedin O. Y., Haensel P., 2001, Phys. Reports, 354, 1 